\title{
Erratum: Achilles' heel of triple negative cancer
}

Erratum

\section{Alberto Ocaña, Juan C. Montero, and Atanasio Pandiella}

Oncoscience. 2014; 1(2): 115-116

PMCID: PMC4278281 PMID: 25594005

Oncoscience. 2014; 1(12): 763-764

PMCID: PMC4303885 PMID: 25621292

Due to the technical error the Editorial "Achilles' heel of triple negative cancer" was repeteadly published in Oncoscience, V1, issue 12, p.763-764.

Original publication was published in Oncoscience, V1, issue 2, p.115-116. 